\title{
Three cage windows for unobstructed animal photography*
}

\author{
WILLIAM K. REDICAN \\ Department of Psychology \\ and \\ California Primate Research Center \\ University of California, Davis, California 95616
}

Three removable cage windows, which make it possible to photograph captive animals with a minimum of obstruction, are described. They are designed for cages with a hinged or sliding door, or as a built-in fixture in the wall of the cage.

The purpose of this paper is to describe three methods for constructing cage windows for unobstructed animal photography, particularly of nonhuman primates.

Many laboratory animals are housed in cages constructed of wire mesh with a hinged front door. In many cases, the animal's temperament does not allow one simply to open the cage door to photograph the S. One alternative is to install a clear acrylite (e.g., Plexiglas) front door. This material, however, is quite easily scratched both by teeth and by metal objects; the scratches are very difficult to remove and invariably intrude into the focal plane. Moreover, if the acrylite door is permanently mounted on the hinges of the cage, it is often difficult to clean accumulated debris from the surface.

One plan for a portable and removable window for a cage with a hinged front door is shown in Fig. 1. It is designed for a cage with an entire front wall that is mounted on two hinges (to the right of an $\mathrm{O}$ facing the S) and can be locked shut with a latch that catches on a projection in the cage frame. In this plan, sheets of acrylite are supported by an aluminum frame of four strips of $3 / 16$-in. (4.8-mm) or $1 / 4-$ in. $(6.4-\mathrm{mm})$ bar stock approximately $1 \mathrm{in}$. in width. These are bolted around the perimeter of the acrylite on the side facing the $S$. To ease alignment of the bolt holes, it is best to drill the frame first, position it on top of the acrylite, and then drill holes through the acrylite. Bolts should be inserted from the S's side, so that the nuts and washers cannot be manipulated by the $S$.

Two layers of acrylite are installed: one $(1 / 8 \mathrm{in}$., or $3.2 \mathrm{~mm}$ ) on the S's side and another ( $3 / 8 \mathrm{in}$., or $9.5 \mathrm{~mm})$ on the O's side. Thus, when the S's side becomes excessively scratched, it can be replaced at far less expense than is required to replace a single thick piece.

The window is mounted onto the existing cage structure by means of three short (2-3 in.) pieces of bar stock bolted onto the window frame on the side facing

*Supported by National Institute of Health Grants MH22253, RR00169, and HD04335, a National Science Foundation traineeship, and funds from the Department of Psychology, University of California, Davis. Reprints may be obtained from William K . Redican Department of Psychology, University of California, Davis, California 95616. I am grateful to Bill Hancock for critically reading this manuscript. the S. Cages are seldom exactly alike, so it is wise to allow for realignment when necessary by drilling extra bolt holes for each bar, spaced at no more than $1 / 4$-in. (6.4-mm) intervals.

Figure $2(a, b)$ illustrates the relationship of the window to the cage frame. With the cage door in an open position, the two mounting bars on the right rest on top of the cage door's hinges; the bar on the left is inserted into the latch restraint of the cage frame. The frame of the open cage door prevents the two bars at the right from slipping off the top of the hinges.

It is generally good procedure, especially with relatively aggressive species such as the rhesus macaque, to proceed slowly and deliberately when inserting and withdrawing the window. It is helpful, but not always necessary, to have a second person standing by to open the cage door and brace it against a possible escape attempt. When the cage door is opened a few inches, the window assembly is introduced from the side, and the door is opened more fully as the mounting bars are positioned both above the hinges and in the latch restraint. (That is, by the time the window is in place, the cage door is swung open fully.) If one proceeds steadily, the probability of a successful escape is low. Perhaps more importantly, since the O's gaze is often a highly arousing stimulus for some monkeys, it is advisable to avoid direct eye contact when the window is either installed or removed. The correctly installed window will withstand a direct assault by a mature male or female macaque.

Some laboratory cages utilize a laterally or vertically sliding door on the front wall. Figure 3 shows a window that is installed over the area covered by this type of access door. The window is secured by two fixed L-shaped upper bars fitted over the existing cage structure and by three lower $1 / 4-\mathrm{in}$. (6.4-mm). thumbscrews tightened against the cage frame. Two layers of acrylite are installed, as in the window unit for the hinged cage, with the thinner layer closer to the $S$. Figure 4 shows the window mounted on a cage with a laterally sliding door.

The window is mounted while the cage door is closed; once the window is in place, the door can be unlocked and moved to the side. It is a good procedure to move the door aside slowly, perhaps initially by only a few inches, to enable the animal to explore the window. When the door is moved aside too abruptly, rhesus macaques, for example, may try to jump through the space left vacant by the door. There is at least one limitation: If a $\mathbf{S}$ positions itself immediately to one side of the window, the animal cannot be photographed without cage bars' being interposed between $S$ and camera.

A third, and perhaps most practical, alternative is 


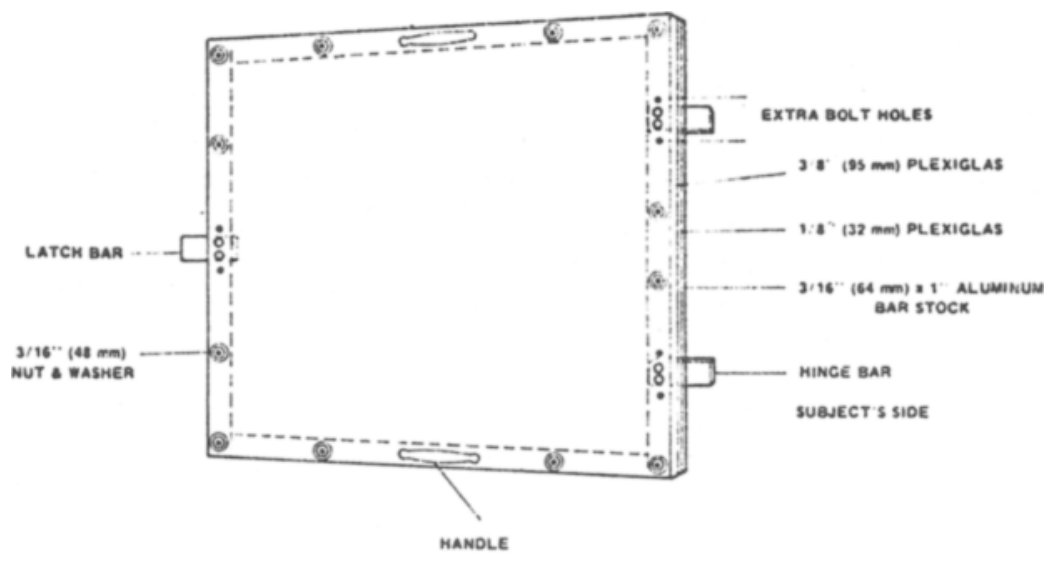

Fig. 1. Window designed for a cage with a hinged front door. All bolts are inserted from the S's side, so that the nuts are not accessible to the $\mathrm{S}$. There are often slight differences in size in the same cage model and the extra bolt holes allow the mounting bars to be readjusted accordingly.

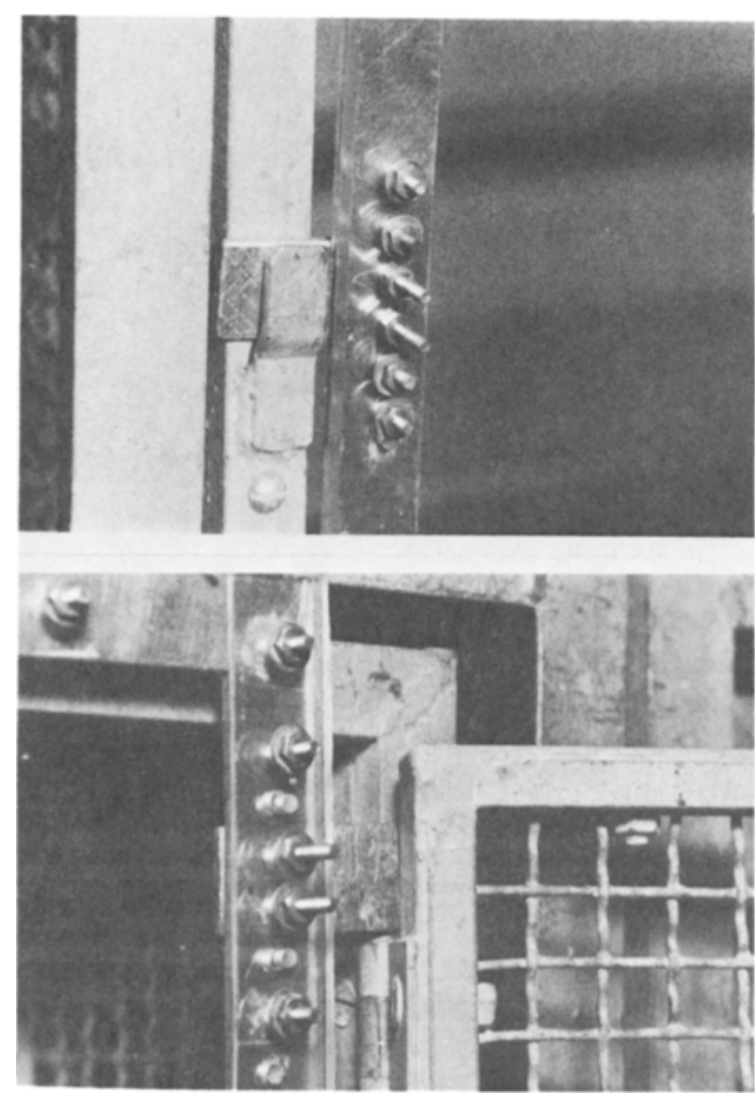

Fig. 2. (a) The bar to the photographer's left is inserted into the latch restraint of the existing cage frame. (b) The bars to the photographer's right are inserted above the hinges of the cage. The window pictured above has been moved slightly to the left to show the position of the bar.

shown in Fig. 5. This window was designed as a permanent wall of a $3 \times 3 \times 3 \mathrm{ft}(91.44-\mathrm{cm})$ cage. It utilizes $1 / 4$-in. (6.4-mm) tempered (not safety plate) glass ${ }^{1}$ which (a) is impossible to scratch except with the hardest of materials, (b) is virtually unbreakable if installed correctly, and (c) is very easily cleaned.

Our first tempered-glass prototype consisted of a

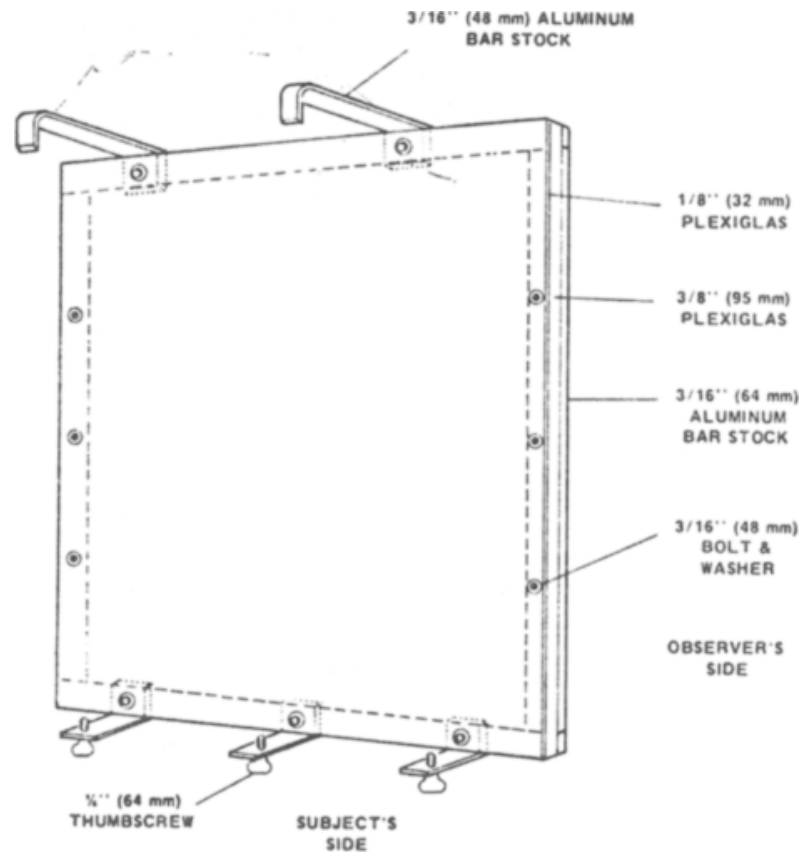

Fig. 3. Window designed for a cage with a sliding front door. The $\mathbf{L}$-shaped projections on top are positioned over a stable bar of the cage frame, and then the three lower thumbscrews are tightened.

single sheet of glass, but Ss dirtied it within moments of being introduced into the cage. A second sheet of glass was subsequently installed and the cage modified so that both panes were removable. When dirty, the inner layer is removed by sliding it to one or, if there is sufficient space, to either side. It is important that means are provided for locking the glass in place to prevent Ss from dislodging it. Edges of the two sheets of glass should be staggered (__ be gripped for withdrawal from the side. Another method is to attach a narrow handle (i.e., no wider than the pane of glass) to the lateral edge of each pane.

Use care during installation. Even though a $1 / 4$-in. $(6.4-\mathrm{mm})$ sheet of tempered glass with an area of $25 \mathrm{ft}^{2}$ $\left(2.32 \mathrm{~m}^{2}\right)$ will withstand a maximum load of $170 \mathrm{lb} / \mathrm{ft}^{2}$, 


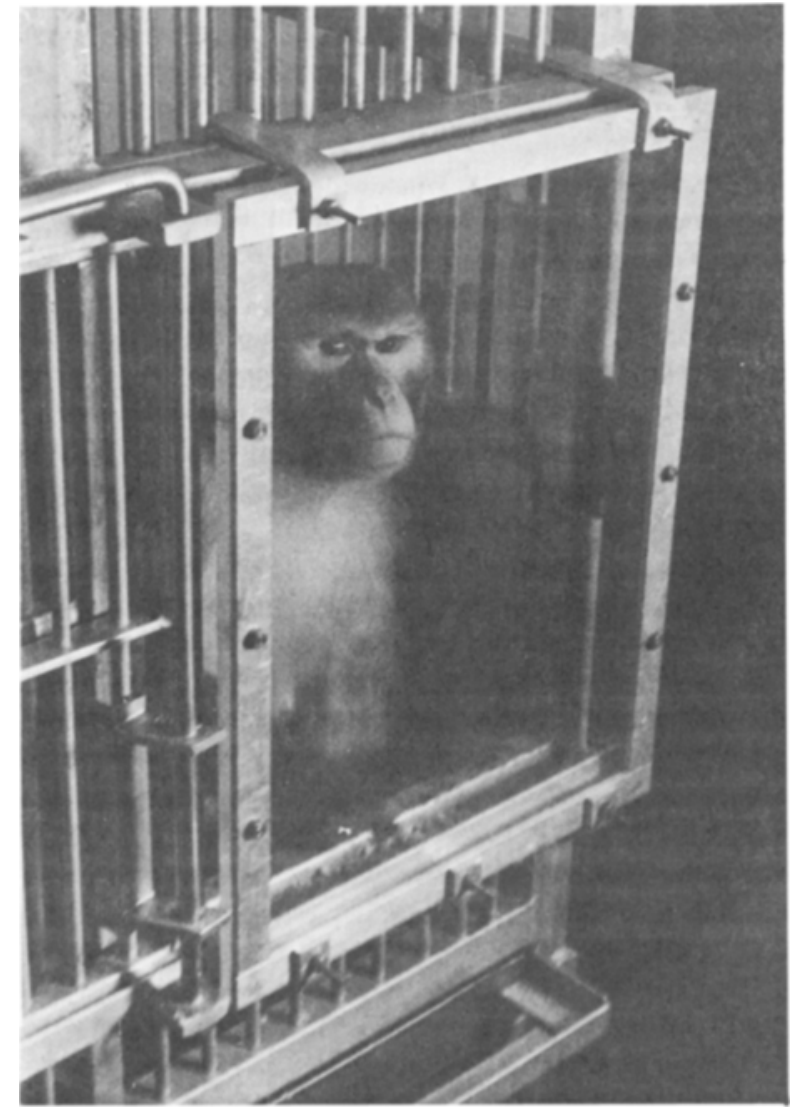

Fig. 4. The second type of window fits over a sliding-rather than hinged-access door of a home cage. An adult male rhesus macaque is shown within.

thus making it 3-5 times stronger than regular glass in sustaining weight and resisting thermal fracture, ${ }^{2}$ a relatively gentle tap on an unprotected edge with a sharp object can shatter an entire tempered sheet. (Unlike other types of glass, tempered glass breaks into small rounded pieces rather than jagged sections, so the likelihood of tissue damage after breakage is minimized.) For.this reason, the glass's edges should be protected by a thin metal frame if possible. Strips of rubber [e.g., B. F. Goodrich's Type II, Grade A, soft, MIL-R6130B, BFG SC41, 3/8 in. $(9.5 \mathrm{~mm})$ thickness] around the perimeter of the cage frame prevent direct metal-to-glass contact and will cushion the glass when the animal jumps against it. One should also take precautions

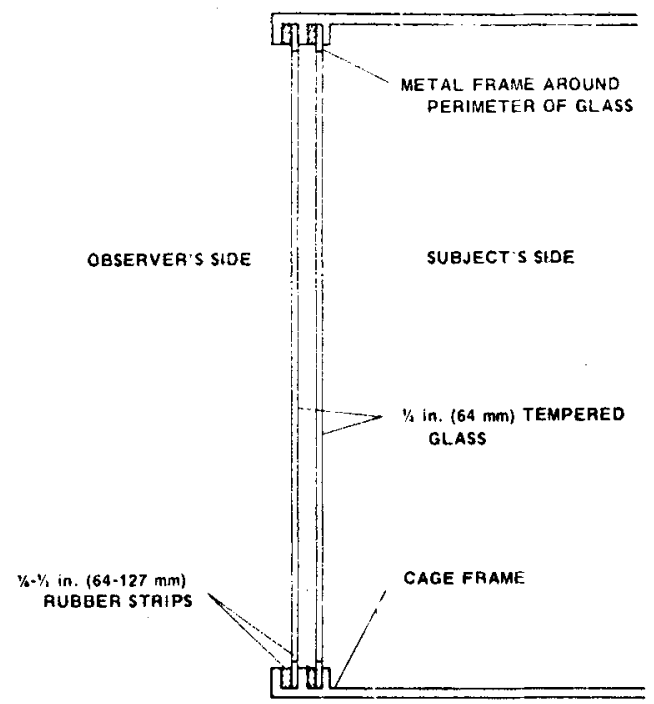

Fig. 5. Wide view of a window designed as an entire permanent wall of a home or test cage.

against exposing the glass to uneven temperature. If, for example, one area of the glass is heated by a floodlight and another is cooled by a draft of air, the glass is more likely to shatter.

The cost of tempered glass varies considerably from dealer to dealer, so it is worthwhile to obtain a number of estimates. A $3 \mathrm{ft} \times 3 \mathrm{ft} \times 1 / 4 \mathrm{in}$. $(91.44 \times 91.44 \times$ $.64 \mathrm{~cm}$ ) piece was purchased for approximately $\$ 25$. Odd sizes are more expensive.

One might expect that glass, rather than acrylite, would be used in the first and second designs discussed in this paper. It is unlikely that the frames suggested for acrylite provide enough structural support for tempered glass. Instead, a stronger frame-which would also protect the edges of the glass-would have to be devised. Tempered glass is appreciably heavier than acrylite, however, and the additional weight of a heavy frame would probably make a portable tempered-glass window rather unmanageable.

\section{NOTES}

1. Leonard A. Rosenblum suggested using tempered glass.

2. Specifications of Libbey-Owens-Ford Company.

(Received for publication December 4, 1972; revision received May 21, 1973.) 